\title{
Comparative effects of two indole-producing Trichoderma strains and two exogenous phytohormones on the growth of Zea mays L., with or without tryptophan
}

\author{
Erick Herrera-Jiménez ${ }^{1}$, Alejandro Alarcón ${ }^{1}$, John Larsen ${ }^{2}$, Ronald Ferrera-Cerrato ${ }^{1 *}$, Serafin \\ Cruz-Izquierdo $^{3}$, Mariana Ronalda Ferrera-Rodríguez ${ }^{1}$
}

${ }^{1}$ Postgrado de Edafología. Colegio de Postgraduados Campus Montecillo. Carretera México-Texcoco Km 36.5. Montecillo, Texcoco, C.P. 56230, Estado de México, México. ${ }^{2}$ Instituto de Investigaciones en Ecosistemas y Sustentabilidad. Universidad Nacional Autónoma de México, Apartado Postal 27-3, CP 58089 Morelia, Michoacán, México. ${ }^{3}$ Postgrado de Recursos Genéticos y Productividad, Colegio de Postgraduados Campus Montecillo. Carretera México-Texcoco Km 36.5. Montecillo, Texcoco, C.P. 56230, Estado de México, México.

*Corresponding author: rferreracerrato@gmail.com

\begin{abstract}
This research evaluated the growth responses of maize plants due to the inoculation of two indole-producing Trichoderma strains in comparison to the application of two exogenous phytohormones. Either T. tomentosum (EMMFRS1C2) or T. harzianum (MichV6S3C2) were inoculated as conidia or as fungal filtrates obtained from Luria-Bertani, liquid medium, with or without the addition of L-Tryptophan $\left(1 \mathrm{~g} \mathrm{~L}^{-1}\right)$. In addition, two concentrations (5 and $10 \mathrm{mg} \mathrm{L}^{-1}$ ) of indole acetic acid (IAA) and indole butyric acid (IBA) from which $2 \mathrm{~mL}$ were applied per plant. Twelve treatments and six replicates distributed in a completely randomized design. Plants were maintained for 50 days in microcosms (perlite and vermiculite, $2: 1 \mathrm{v} / \mathrm{v})$, under greenhouse conditions $\left(38^{\circ} \mathrm{C} \pm 5\right.$ ) and watered every $48 \mathrm{~h}$. The best plant growth responses (height, root length, leaf area, and root, stem and total dry weight were achieved by inoculating conidia of either T. tomentosum (EMMFRS1C2) or T. harzianum (MichV6S3C2) in combination with tryptophan $\left(1 \mathrm{~g} \mathrm{~L}^{-1}\right)$ when compared to control plants; but $T$. harzianum (MichV6S3C2) only resulted in beneficial effects in root length. Moreover, the application of fungal filtrates had similar plant growth responses than the application of either IAA or IBA at $5 \mathrm{mg} \mathrm{L}^{-1}$.
\end{abstract}

Keywords: IBA, IAA, vegetative growth, root system, tryptophan, conidia, fungal filtrates 


\section{Introduction}

The genus Trichoderma (Teleomorph Hypocrea, Ascomycota, Dikarya) includes a diverse and soil-borne cosmopolitan species interacting with microbial communities (Martínez-Peña et al., 2016; Arteaga-Garibay et al., 2016). Some species are saprobes while others have been detected within the plant roots, acting as nonvirulent opportunistic endophytes (Mastouri et al., 2010). Moreover, the Trichoderma species play an important role in the health of an ecosystem (Rajput et al., 2014a). Since the 1930s, the Trichoderma species are recognized and used as biological control agents directed for reducing the severity fungal diseases in plants (Colla et al., 2015). Currently, some studies are focused on evaluating the positive effects of Trichoderma species as plant growth promoters and as crop yield stimulators (Mastouri et al., 2010; Lorito et al., 2010). Other beneficial effects of Trichoderma are related to the degradation of toxic compounds, to the stimulation of root development due to the production of phytoregulators, to the increase of solubilization of insoluble forms of phosphorus $(\mathrm{P})$ in soil, and to act as P-mobilizing microorganisms for plants (Contreras-Cornejo et al., 2014a).

The positive Trichoderma-plant interaction is mutually linked to those carbon sources released due to root exudation into adjacent soil (rhizosphere), which stimulate the growth and proliferation of several fungi, including Trichoderma species (Amore et al., 2013). For example, plant-derived sucrose is an important carbon source facilitating the colonization of Trichoderma in the root system, and then, inducing relevant beneficial effects in plants (Vargas et al., 2009). In this regard, Vos et al. (2015) indicate that Trichoderma species have diverse mechanisms for improving plant growth, and furthermore, information is focused on the production of beneficial volatile compounds (Lee et al., 2016). For example, T. viride stimulated the growth of Arabidopsis thaliana without being in physical contact with the root system, but inducing greater formation of lateral roots and stimulating early flowering (Hung et al., 2013). Likewise, T. harzianum release volatile antibiotic compounds against pathogenic fungi, which also act as molecules for stimulating the growth of Solanum lycopersicum (Vinale et al., 2008). Trichoderma species improve plant growth and development under in vitro systems, greenhouse conditions, or field conditions. Several studies have proven the beneficial effects of Trichoderma spp. on vegetable crops like cucumber, periwinkle, chrysanthemum, and lettuce, by stimulating seed germination, vegetative or reproductive growth (Studholme et al., 2013).

The efficacy of Trichoderma as a biofertilizer agent has been proven when fungal species were applied to soil, seeds, or plants, and their benefits are related to increased nutrient solubility, as well as to improved nutrient uptake by roots, likewise the mode of action of plant growth promoting bacteria (Molla et al., 2012; Zhao et al., 2014; Martínez et al., 2015).. Thus, Trichoderma strains may help farmers for improving the use efficiency of fertilizers, then minimizing the potential negative effects of fertilizers in the environment (Rajput et al., 2014b). For instance, Molla et al. (2012) proved that supplementing fertilizers in combination with the inoculation of Trichoderma resulted in enhanced yield (over $50 \%$,) of tomato plants when compared to the sole application of NPK. 
On the other hand, auxins were identified as phytohormones because of their ability for stimulating plant growth in response to light stimuli. Applications of IAA or synthetic auxins may induce significant changes in plant growth and development (Bonner and Bandurski, 1952). Much of the knowledge about the physiological functions of auxins in plants is based from studies in which the evaluation of plant responses in the presence of phytohormone precursors (Vanneste, and Friml, 2009), but no information is available when Trichoderma strains are combined.

Thus, this work evaluated the effect of two indoleproducing Trichoderma strains on the growth of maize plants, when compared to effects due to the application of two growth regulators, and to the addition of L-Tryptophan as an indole precursor.

\subsection{Materials and Methods}

\subsection{Biological material}

Two strains of Trichoderma ( $T$. tomentosum EMMFRS1C2, and T. harzianum MichV6S3C2) previously isolated from roots of maize plants grown at field conditions (unpublished data) were used for this research. Previous experiments demonstrated that these two fungal strains, over 60 Trichoderma strains tested, were able to produce indole-type compounds under in vitro systems; this indole compounds production was as follows: 0.55 $\mu \mathrm{g} \mathrm{mL}^{-1}$ for $T$. harzianum, and $2.430 \mu \mathrm{g} \mathrm{mL}^{-1}$ for $T$. tomentosum (Herrera-Jiménez, unpublished data).

In addition, seeds of maize CP-V20 were surface disinfected with sodium hypochlorite $2 \%$ for $5 \mathrm{~min}$ in constant agitation, rinsed three times with sterile distilled water, dried with sterile absorbent paper, and placed in sterile flasks until utilization.

\subsection{Preparation of the fungal filtration and} production of spores used as inoculum

The two Trichoderma strains were individually placed in Luria-Bertani liquid medium $\left(\mathrm{g} \mathrm{L}^{-1}: 10\right.$ bacto tryptone DIFCO ${ }^{\circledR}, 5$ yeast extract, $5 \mathrm{NaCl}, \mathrm{pH}$ adjusted to 7.5 with $1 \mathrm{~N} \mathrm{NaOH}$ before autoclaving; Bric et al., 1991), with and without L-Tryptophan $\left(1 \mathrm{~g} \mathrm{~L}^{-1}\right)$ and incubated at $28^{\circ} \mathrm{C}$ for $48 \mathrm{~h}$ in agitation at $160 \mathrm{rpm}$. Subsequently, each fungal strain was filtered with a millipore membrane $(0.22 \mu \mathrm{m}$, Millex $\left.{ }^{\circledR}\right)$. The filtrate was individually placed in $50 \mathrm{~mL}$ flasks until its utilization.

Both fungi were grown in potato-dextrose-agar (PDA, Merck ${ }^{\circledR}$ ) medium at $28{ }^{\circ} \mathrm{C}$ in total darkness for $48 \mathrm{~h}$. Subsequently, both strains were exposed to light for stimulating conidia production.

The preparation of the Trichoderma spore suspension was done under sterile conditions, using Petri dishes with fungal growth in PDA at $28^{\circ} \mathrm{C}$ for $72 \mathrm{~h}$. Moreover, $15 \mathrm{~mL}$ of sterile distilled water and a drop of Tween 20 were added to each Petri dish that was gently stirred. The resulted conidia suspension was collected and placed in beakers with $40 \mathrm{~mL}$ of sterile distilled water and one drop of Tween 20. The colony forming units (CFU) estimation for each fungal strain was performed by preparing serial dilutions based on the methodology of Madigan et al. (2003). One milliliter of fungal suspension taken and placed in a test tube with $9 \mathrm{~mL}$ of sterile distilled water, after which dilutions were made from $10^{-1}$ to $10^{-8}$. Prior to plating, each of the dilutions were homogenized with the aid of a Vortex agitator (EG Technic). Then, $100 \mu \mathrm{L}$ were placed and distributed on PDA contained in Petri dishes by triplicate, and incubated at $28{ }^{\circ} \mathrm{C}$ in complete darkness for $48 \mathrm{~h}$; then, the CFU were recorded. 


\subsection{Establishment of the experiment}

Experiment was conducted under controlled conditions to assure the beneficial effects of fungal strains with or without L-Tryptophan in comparison to the application of both target auxins. Thus, a 22-cm high x $29 \mathrm{~cm}$ long x $2 \mathrm{~cm}$ wide microcosm with three compartments was used. Each microcosm was filled with $10 \mathrm{~g}$ of substrate (vermiculite+perlite $\mathrm{v} / \mathrm{v}$ ). Three maize seeds were sown in each compartment, and microcosms were irrigated with distilled water every $24 \mathrm{~h}$ to allow seed germination. Each plant at the corresponding treatment was inoculated at V2 stage (15 days after sowing) with $2 \mathrm{~mL}$ of the filtered product of each fungal strain, or with $2 \mathrm{~mL}\left(1 \times 10^{8} \mathrm{CFU} \mathrm{mL}\right.$ $\left.{ }^{1}\right)$ of the conidia suspension collected from each Trichoderma species.

In addition, to compare the effects of fungal treatments, other set of plants were subjected to the application of two growth regulators (Sigma-Aldrich ${ }^{\circledR}$ ), indole-butyric acid (IBA) and indole-acetic acid (IAA), which were applied at two concentrations ( 5 and $10 \mathrm{mg} \mathrm{kg}^{-1}$ ) to the corresponding treatments. In addition, plants with the application of L-Tryptophan as well as the inclusion of an absolute control, and plants inoculated with both Trichoderma strains with and without L-Tryptophan, were included as experimental treatments (six replicates per treatment).

Plants were kept under greenhouse conditions (average temperature from $28-30{ }^{\circ} \mathrm{C}$, and average relative humidity from $30-40 \%$ during the day), and irrigated with distilled water every $24 \mathrm{~h}$ for maintaining field capacity in the substrate. The experiment was performed in duplicate to confirm the effects obtained from the first assay.

\subsection{Evaluation of the variables}

Since either the inoculation of Trichoderma or the application of IAA and IBA were meant to evaluate their effects on plant growth promotion, we select the following growth parameters for validating our hypothesis under our experimental conditions. After 30 days, plants were harvested to evaluate plant height, root length, and leaf area (LI-3100C Area Meter, USA) by placing leaves in a corrugated paper press until measurements. The total dry weight was also determined by placing plant tissue in perforated paper bags at $70{ }^{\circ} \mathrm{C} \pm 2$ ${ }^{\circ} \mathrm{C}$ for $72 \mathrm{~h}$ (FELISA, model 242-A) and weighed in an analytic balance (Sartorius, analytic AC $210 \mathrm{~S}$ ). In addition, the chlorophyll content was determined through the greenness index expressed in SPAD units (MCL502 Minolta SPAD 502), and the photochemical efficiency $(\mathrm{OS} 30 \mathrm{p}+)$ was measured by considering the fourth mature leaf. Fungal population (CFU) was estimated by taking $10 \mathrm{~g}$ of substrate from those treatments inoculated with both Trichoderma strains. This fungal population was evaluated by following the serial dilution and plate counting technique (Madigan, 2003), considering three replicates per treatment $(n=3)$. From the corresponding fungal dilutions, $100 \mu \mathrm{L}$ were taken, and uniformly distributed on the surface of PDA + Rose of Bengal contained in Petri dishes. The Petri dishes were incubated at 28 ${ }^{\circ} \mathrm{C}$ for $48 \mathrm{~h}$, and fungal $\mathrm{CFU}$ were recorded. Then, data were transformed into base 10 logarithms for performing the respective statistical analysis.

\subsection{Molecular identification of Trichoderma strains}

The two Trichoderma strains were subjected to the molecular identification at the Instituto de Biología 
with the supervision of Dr. Roberto Garibay Origel during a workshop for microbial molecular identification. The DNA extraction was performed with the XNAP kit (Sigma-Aldrich ${ }^{\circledR}$ ) for obtaining a high-quality DNA suitable for PCR amplification. For identification the internal transcript spacer (ITS 1, ITS2) regions were amplified by means of the polymerase chain reaction (PCR) in $20 \mu \mathrm{L}$ reaction in which the following primers were utilized ITS1F (TCC GTA GGT GAA CCT GC GG) and ITS4R (TCC TCC GCT TAT TGA TAT GC) $(0.2 \mu \mathrm{M})$. PCRcycle consisted on initial denaturalization $94{ }^{\circ} \mathrm{C} 2$ min, 35 cycles $\left(94{ }^{\circ} \mathrm{C} 1 \mathrm{~min}, 51^{\circ} \mathrm{C} 1 \mathrm{~min}, 72{ }^{\circ} \mathrm{C}\right.$ ) with a final extension of $8 \mathrm{~min} 72^{\circ} \mathrm{C}$ (Schoch et al., 2012). PCR products were verified with electrophoresis in agarose gel (80v $60 \mathrm{~min})$ and purified with EXOSapit (Affymetrix). Sequences were obtained by Sanger sequencing. Bioinformatic analysis were performed using Genious software, and the fungal identity was assigned using BLAST (Basic Local Alignment Search Tool), to depict the phylogenetic relationship of the two fungal strains used for the study, and the closest homologous sequences were retrieved from NCBI GenBank.

\subsection{Statistical analysis}

A completely randomized design was used including 14 treatments with six replicates, yielding 84 experimental units. Data were subjected to the analysis of variance (ANOVA) and the mean comparison test (LSD, $\alpha=0.05$ ). Data were expressed as mean and the mean standard error. Significant differences in all growth variables were analyzed by analysis of variance (ANOVA) to compare the treatment effects of the two fungal species with the application of L-Tryptophan and the comparison to the application of the two exogenous auxins. Statistical analyses were performed using SAS 9.0 and SigmaPlot 11.0.

\section{Results}

\subsection{Effects on plant height and root length}

Figure 1 shows a general view of plant growth responses due to the application of phytohormones or the inoculation of both fungal strains with or without the addition of tryptophan. The greatest plant height was obtained by inoculating conidia of both Trichoderma strains plus tryptophan and by applying $5 \mathrm{mg} \mathrm{IBA} \mathrm{kg}^{-1}$ when compared to the control (Figure 2a). Furthermore, the combination of conidia of MichV6S3C2 (T. harzianum) plus tryptophan had positive effects on the root length when compared to the control, and to treatments with the application of each plant growth regulator, excepting with IAA at $5 \mathrm{mg} \mathrm{kg}^{-1}$ (Figure 2b).

\subsection{Effects on the leaf area and on greenness index (SPAD units)}

The inoculation of either $T$. tomentosum (EMMFRS1C2) or T. harzianum (MichV6S3C2) combined with tryptophan resulted in significantly greater leaf area when compared to control plants (Figure 3a). Moreover, the application of the filtrate of T. harzianum without tryptophan had similar effects on the leaf area in comparison to the application of $5 \mathrm{mg} \mathrm{IBA} \mathrm{kg}{ }^{-1}$; however, these treatments showed significant differences in respect to control plants (Figure 3a).

The greenness index (SPAD units) was significantly greater in plants inoculated with conidia of $T$. harzianum (MichV6S3C2) without tryptophan when compared to those plants inoculated with conidia of T. tomentosum (EMMFRS1C2) without the addition of tryptophan (Figure 3b). The application of each phytohormones did not showed significant differences in respect to control plants (Figure 3b). 


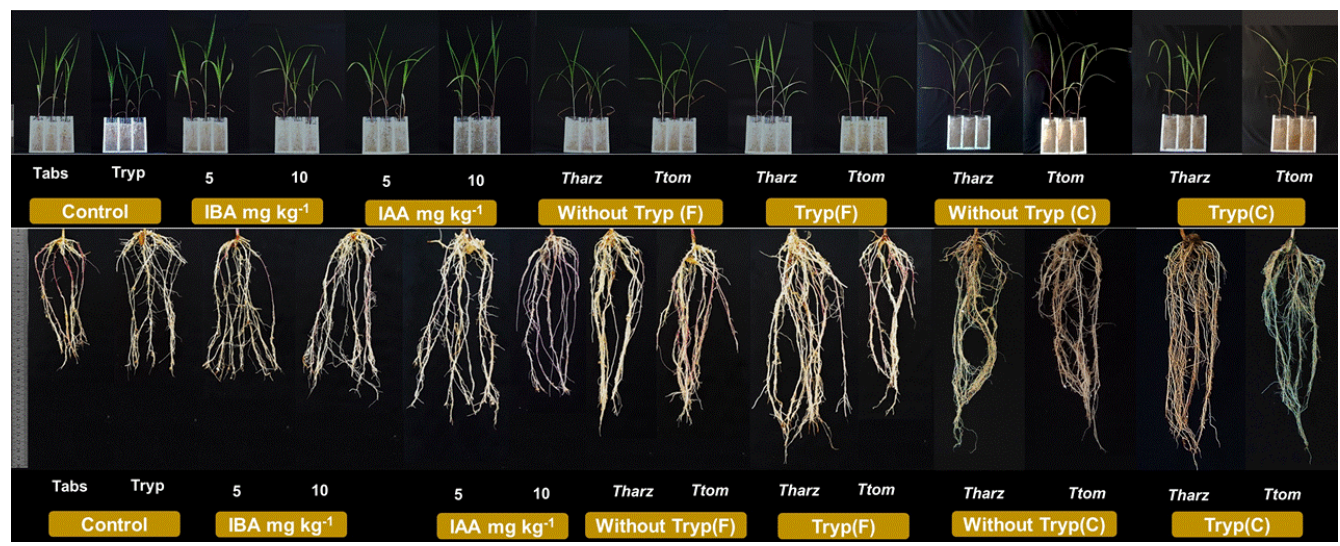

Figure 1. Growth responses of Zea mays L. plants inoculated with conidia or with fungal filtrates of two strains of Trichoderma, in comparison to the effects obtained by the application of two exogenous phytohormones, and the application of L-Tryptophan, after 30 days of growth. Abbreviations: Tabs: Control; IBA: indole butyric acid (5 or 10 $\mathrm{mg} \mathrm{kg}^{-1}$ ); IAA: indole acetic acid (5 or $10 \mathrm{mg} \mathrm{kg}^{-1}$ ); Tryp: L-Tryptophan; Ttom: strain EMMFRS1C2 (T. tometosum); Tharz: strain MichV6S3C2 (T. harzianum); (F): fungal filtrate from liquid media culture; (C): conidia suspension. $\mathrm{n}=6$.
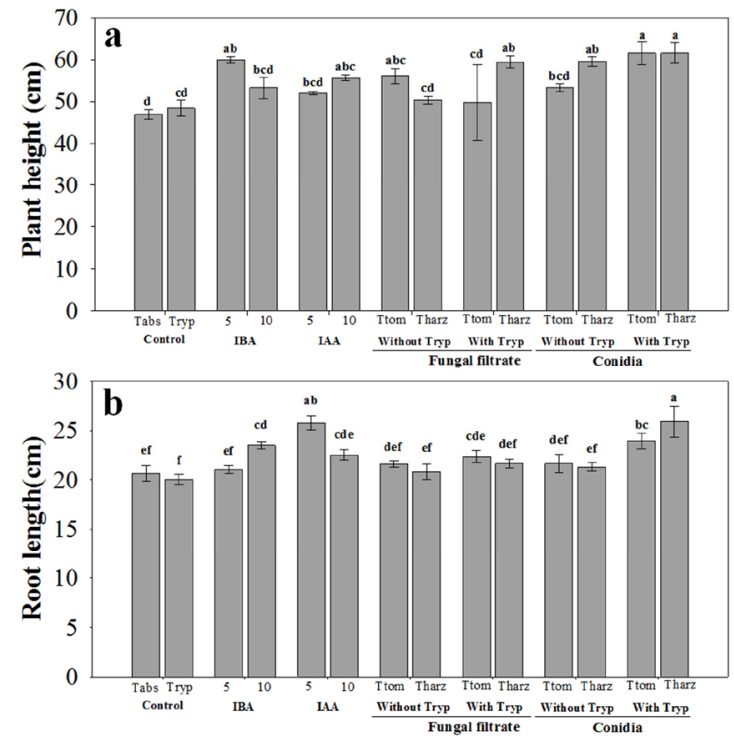

Figure 2. Height (a) and root length (b) of Zea mays L. plants inoculated with conidia or with fungal filtrates of two strains of Trichoderma, in comparison to the effects obtained by the application of two exogenous phytohormones, and the application of L-Tryptophan, after 30 days of growth. Abbreviations: Tabs: Control; IBA: indole butyric acid (5 or $10 \mathrm{mg} \mathrm{kg}^{-1}$ ); IAA: indole acetic acid (5 or $10 \mathrm{mg} \mathrm{kg}^{-1}$ ); Tryp: L-Tryptophan; Ttom: strain EMMFRS1C2 (T. tometosum); Tharz: strain MichV6S3C2 (T. harzianum); (F): fungal filtrate from liquid media culture; (C): conidia suspension. $\mathrm{n}=6$. Means with the same letter are not significantly different (LSD, $\alpha=0.05)$. 

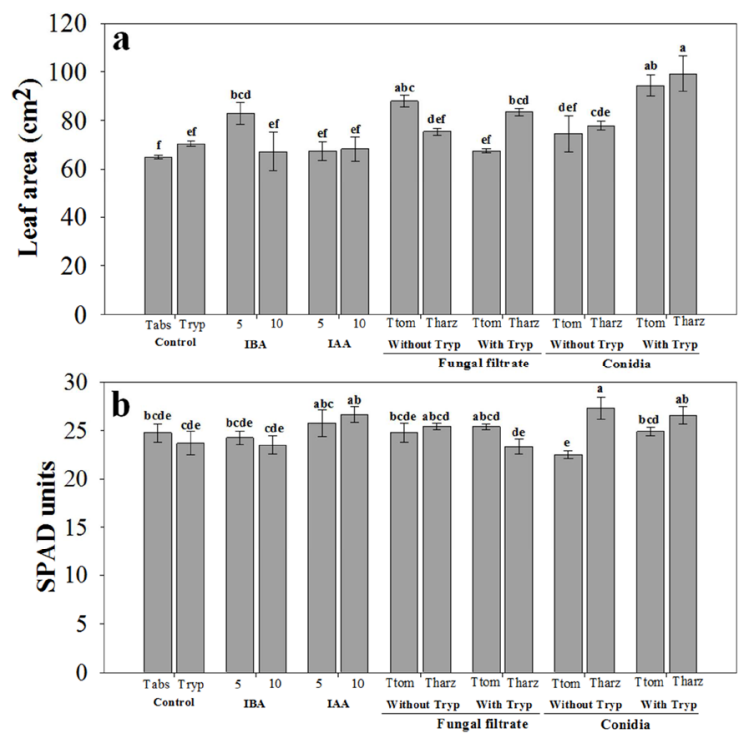

Figure 3. Leaf area (a) and SPAD units (b) of Zea mays L. plants inoculated with conidia or with fungal filtrates of two strains of Trichoderma, in comparison to the effects obtained by the application of two exogenous phytohormones, and the application of L-Tryptophan, after 30 days of growth. Abbreviations: Tabs: Control; IBA: indole butyric acid (5 or $10 \mathrm{mg} \mathrm{kg}^{-1}$ ); IAA: indole acetic acid (5 or $\left.10 \mathrm{mg} \mathrm{kg}^{-1}\right)$; Tryp: L-Tryptophan; Ttom: strain EMMFRS1C2 (T. tometosum); Tharz: strain MichV6S3C2 (T. harzianum); (F): fungal filtrate from liquid media culture; (C): conidia suspension. $\mathrm{n}=6$. Means with the same letter are not significantly different (LSD, $\alpha=0.05)$.

\subsection{Effects on dry matter accumulation}

Accumulation of plant dry matter is shown in Figure 4. The root dry weight was significantly enhanced due to the inoculation of conidia from EMMFRS1C2 ( $T$. tomentosum) added with tryptophan in comparison to control plants. Moreover, the application of filtrates from $T$. harzianum without tryptophan or from $T$. tomentosum plus tryptophan had similar responses on root length when compared to the application of $5 \mathrm{mg} \mathrm{IBA} \mathrm{kg}{ }^{-1}$, but significantly greater than control plants (Figure 4a).

The stem dry weight was significantly enhanced when plants were inoculated with conidia from each fungal strains but when tryptophan was added (Figure 4b). In addition, the application of $5 \mathrm{mg}$ IBA kg-1 resulted in significantly greater stem dry weight than control plants (Figure 4b). The leaf dry weight was significantly stimulated when plants were inoculated with conidia from T. harzianum without or with tryptophan when compared to the application of 5 mg IAA kg-1 , but these three treatments did not show significant differences when compared to control plants (Figure 4c)

Plants either inoculated with conidia from $T$. harzianum or T. tomentosum in combination with tryptophan showed the greatest accumulation of total dry weight in comparison to control plants (Figure 4d). Furthermore, the application of $5 \mathrm{mg}$ IBAkg $^{-1}$ resulted in significant improved total dry weight when compared to plants with IAA the application or control plants (Figure 4d). 

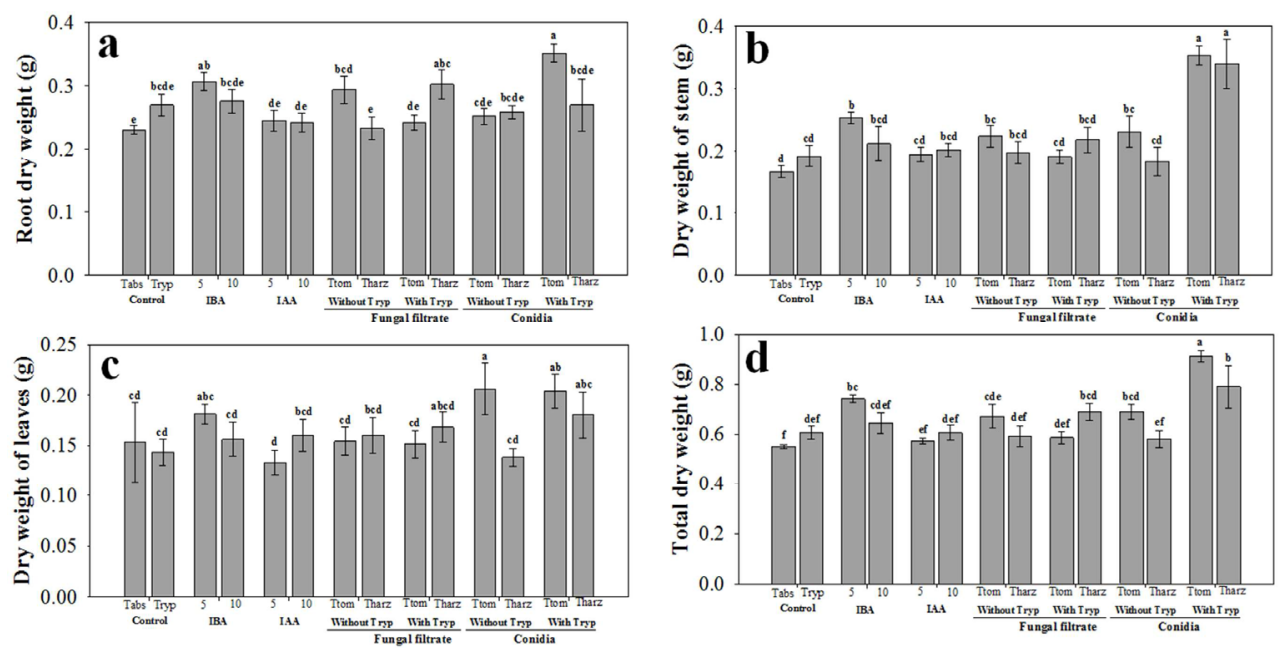

Figure 4. Root dry weight (a), dry weight of stem (b), dry weight of leaves (c) and total dry weight of Zea mays L. plants inoculated with conidia or with fungal filtrates of two strains of Trichoderma, in comparison to the effects obtained by the application of two exogenous phytohormones, and the application of L-Tryptophan, after 30 days of growth. Abbreviations: Tabs: Control; IBA: indole butyric acid (5 or $\left.10 \mathrm{mg} \mathrm{kg}^{-1}\right)$; IAA: indole acetic acid (5 or $10 \mathrm{mg} \mathrm{kg}^{-1}$ ); Tryp: L-Tryptophan; Ttom: strain EMMFRS1C2 (T. tometosum); Tharz: strain MichV6S3C2 (T. harzianum); (F): fungal filtrate from liquid media culture; $(\mathrm{C})$ : conidia suspension. $\mathrm{n}=6$. Means with the same letter are not significantly different (LSD, $\alpha=0.05$ ).

The number of CFU of Trichoderma $\mathrm{g}^{-1}$ of soil was lower for the treatment inoculated with conidia suspension of $T$. tomentosum plus tryptophan (1.03 $\log _{10}$ ) when compared to the treatment with $T$. harzianum without tryptophan $\left(1.16 \log _{10}\right)$. On the other hand, plants inoculated with $T$. tomentosum without tryptophan $\left(1.13 \log _{10}\right)$ and those with $T$. harzianum with tryptophan $\left(1.06 \log _{10}\right)$ did not show significant differences (Figure 5).

\subsection{Identity assignation for Trichoderma strains}

Strains were identified as Trichoderma harzianum (MichV6S2C3) and Trichoderma tomentosum (EMMFRS1C2) with a $99-100 \%$ of identity. Sequences were submitted to GenBank database, and the accession number assigned corresponded to MF470187 for T. tomentosum (EMMFRS1C2), and MF470188 for T. harzianum (MichV6S2C3). 


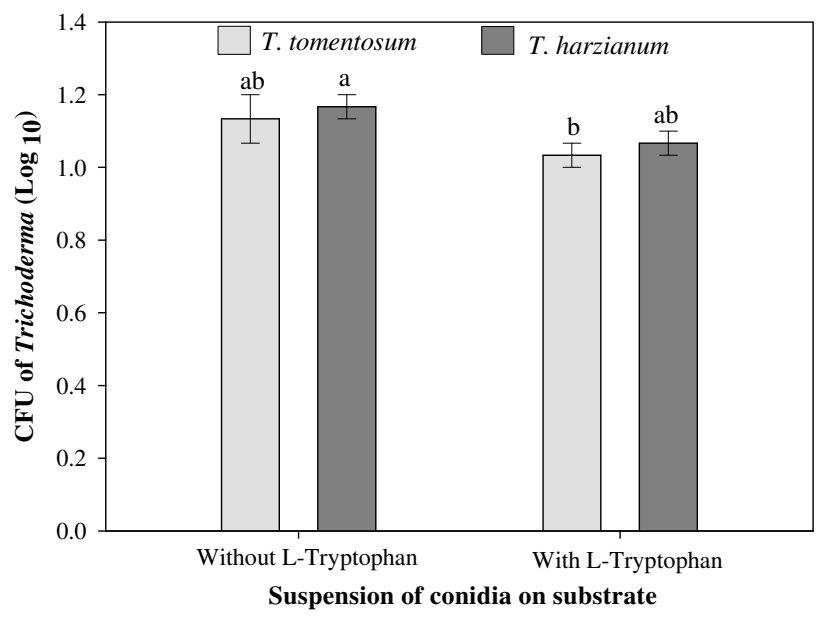

Figure 5. Colony forming units (CFU) of two strains of Trichoderma (T. tomentosum, T. harzianum) in the rhizosphere of maize plants, with and without L-Tryptophan, after 30 days of growth. Means with the same letter are not significantly different (LSD, $\alpha=0.05) . \mathrm{n}=3$.

\section{Discussion}

The present results denoted that the effect of each Trichoderma strain had on maize plants can be attributed to their indole production which act as plant growth stimulator; thus, the beneficial effects achieved by inoculating fungal conidia in plants was reflected in improved root architecture (root length), leaf area and total dry matter. The later concurs with those effects reported by Rubio et al. (2017) who mentioned that $T$. harzianum increased root length and dry weight, as well as height, leaf number and dry weight, leaf area, stem diameter, and number of flowers in tomato plants. However, these effects may be regulated by the fungal genotype, as well as by its origin. Bjorkman et al. (1998) stated that T. harzianum T22 induced growth promotion in maize plants when successfully established in the rhizosphere. Our results indicate that the inoculation of T. harzianum or T. tomentosum had variations on stimulating certain growth parameters in maize plants even though they were inoculated under controlled conditions. Thus, it is possible to mention the existence of certain compatibility between Trichoderma strains and plants by which their effectiveness on plant growth may be dependent.

In addition, some research has been focused on the evaluation of the effectiveness of applying exogenous sources of plant growth phytoregulators, as well as inoculating some microorganisms able to produce such phytohormones (Contreras-Cornejo et al., 2014a; Contreras-Cornejo et al., 2014b). For instance, several species of Trichoderma may stimulate plant growth; then, a hypothesis has emerged in regards of the ability of such fungi for releasing or inducing the synthesis of phytoregulators (Studholme et al., 2013). This may explain, in part, why in our experimental conditions the conidia inoculation of both Trichoderma strains or the application of fugal filtrates resulted in positive responses on plant growth. Moreover, the Trichoderma effects are compared to those obtained due to the application of low doses $\left(5 \mathrm{mg} \mathrm{L}^{-1}\right)$ of IBA 
or IAA, as well as to the effect obtained by the addition of L-Tryptophan. In this regards, the application of conidia was based on the evaluation of the presence of either fungus in the root systems and on the plant growth; but the application of the fungal filtrated was based on the evaluation of potential secondary metabolites (for instance, total indole compounds) that may cause modifications on plant growth, as observed for the sole application of the two doses of either IAA or IBA in the root systems of maize.

The most studied Trichoderma species in regards to their stimulation on growth of several vegetable crops are $T$. asperellum, T. atroviride, T. harzianum, $T$. virens, and T. viride. For example, the mix of three Trichoderma species (T. harzianum, T. viride, and $T$. virens) increased plant height and total biomass, and improved $\mathrm{P}$ and $\mathrm{N}$ uptake in chickpea shoots and roots, under greenhouse and field conditions (Saravanakumar et al., 2013).

The application of Trichoderma based inocula in early plant stages may allow maximizing potential benefits on root development and nutrient absorption (Rajput et al., 2014a). Nevertheless, their effects may be linked to diverse and complex fungus-plant signaling, which may result in specific plant-microorganism affinities and effects (Molla et al., 2012).

The important role of Trichoderma spp. in improving soil fertility and plant growth is due to its capacity to transform organic sources into inorganic available forms, including phosphorus, nitrogen, and iron, which are similar effects to P-solubilizing bacteria; however, the mechanisms of action are different (Saravanakumar et al., 2013; Zhao et al., 2014; Menezes-Blackburn et al., 2016). Then, nutrient solubilization by Trichoderma spp. is especially relevant under reduced soil fertility conditions; thus, if soil fertility is optimal, the chances for obtaining benefits on plant growth due to Trichoderma inoculation may be significantly reduced (Contreras-
Cornejo et al., 2014; Tucci et al., 2011). On the other hand, if plants are grown under suboptimal conditions (temperature, light, water, and nutrient limitation), the inoculation of Trichoderma may have the opportunity for promoting plant growth (Tucci et al., 2011). For example, seedlings inoculated with $T$. longipile under greenhouse conditions showed better growth responses when compared to plants growing at optimal light conditions; the latter denotes those variations of Trichoderma species on plant growth effectiveness depending on environmental conditions (Schmoll et al., 2010). Akladious and Abbas (2012) also obtained growth promotion in corn plants due to the inoculation of Trichoderma, especially when inoculated in less vigorous plants.

The effectiveness of inoculating Trichoderma strains or species may be a result of the affinity/compatibility with the plant genotype (Mastouri et al., 2010; Tucci et al., 2011). For example, the inoculation of six Trichoderma strains had consistent beneficial effects on promoting the growth of lettuce and cucumber plants (Colla et al., 2015); however, the inoculation of a single species resulted in less benefit on the growth of soybean and radish plants (Carvalho et al., 2011). In the present study, we expected differential effects due to the type of the inoculum of Trichoderma (fungal filtrates or conidia suspension), and furthermore, due to the application of L-Tryptophan as an inductor the production of indole-type compounds.

The inoculation of Trichoderma species does not always reflect in beneficial effects on plant growth (Mastouri et al., 2010; Colla et al., 2015). The later may be explained due to the inoculum formulation, which may drastically affect their capability for colonizing the plant root system (Mastouri et al., 2010; Zhao et al., 2014). Moreover, the effects of Trichoderma for stimulating or inhibiting the plant growth may be also the result of the release of secondary metabolites (Azarmi et al., 2011; Rubio et al., 2014). In the 
present study, either T. harzianum or T. tomentosum has differential responses on maize plants, and more importantly, these effects were improved when L-Tryptophan was applied in the inoculated plants. The latter indicates that some Trichoderma strains may need the presence or synthesis of phytohormones inducers for improving their ability of promoting the plant growth. However, further studies are needed for identifying external factors (for instance, soil type, fertilization, nutritional status of plants, etc.) that may affect the potential benefits of indole-producing Trichoderma strains on plant growth.

The benefits on plant growth due to the inoculation of Trichoderma spp., has been demonstrated for maize plants under field conditions and under several biotic and abiotic interactions (Mastouri et al., 20010; Akladious and Abbas, 2012; Kumar et al., 2016). However, these fungal inoculations have shown differential variations on plant growth depending on the presence of several limiting factors such as location, seasonality, crop genotype, and type and formulation of the fungal inoculum (Colla et al., 2015; Akladious and Abbas, 2012; Rajput et al., 2014a; Rajput et al., 2014b).

The microbial interactions can be beneficial, harmful, or neutral, and they may significantly influence plant growth and development, also these interactions may favor positive plant responses depending on the relations between fungi and endophytic bacteria (Saravanakumar et al., 2013; Gupta et al., 2016). The effects obtained from both Trichoderma strains in maize plants in the present research, denote the potential of these fungal strains for inducing plant growth promotion since both fungal species are able to synthesize and release indole-type compounds under in vitro cultures. Moreover, this fungal ability is improved when the L-Tryptophan a precursor of indoletype compounds, was combined to the inoculated plants. The latter indicates that it is necessary to make a careful screening of beneficial Trichoderma strains, and their combination with the application of indole-precursors.

More importantly, Trichoderma species may release soluble compounds in the culture media that may potentially exert beneficial effects not only for plants but also for those microbial groups cohabiting the rhizosphere (Zeilinger et al., 2016). In this regards, the application of fungal extracts resulted in similar effects on plant growth to those obtained when either IBA of IAA were applied in plants at the lowest dose $\left(5 \mathrm{mg} \mathrm{L}^{-1}\right)$. Nevertheless, further studies are needed for identifying those soluble fungal metabolites, which may be extracted and applied in agricultural crops for inducing better growth and vigor.

\section{Conclusions}

The inoculation of both Trichoderma strains ( $T$. tomentosum EMMFRS1C2 and T. harzianum MichV6S3C2) showed beneficial effects on promoting growth in maize plants. Tryptophan as an IAA precursor also resulted in beneficial effects, especially when applied to plants inoculated with the conidia suspension, which resulted in increased plant height and root length. The application of IBA or IAA in plants resulted in greater development of root systems when compared to the effect of both Trichoderma strains. The application of IBA or IAA did not result in greater plant growth when compared to the effects obtained with Trichoderma strains; however, a similar effect was observed for root development in comparison to the inoculation of both Trichoderma strains. On the other hand, the addition of L-Tryptophan showed significant effects on plant growth promotion. 


\section{Acknowledgments}

This work was financially supported by the SEPCONACYT grant 2012-179319. Special thanks to the National Council of Science and Technology (CONACYT-Mexico) for financial support provided to E.H-J during his master degree program. Special thanks for two anonymous reviewers whose comments contributed on improving this manuscript.

\section{References}

Akladious, S. A and Abbas S. M. 2012. Application of Trichoderma harzianum T22 as a biofertilizer supporting maize growth. African Journal of Biotechnology. 11, 8672-8683.

Amore, A., Giacobbe, S and Faraco, V. 2013. Regulation of cellulase and hemi-cellulase gene expression in fungi. Curr. Genomics.14, 230-249.

Amore, A., Giacobbe, S. and Faraco, V. (2013) Regulation of cellulase and hemi-cellulase gene expression in fungi. Curr. Genomics.14, 230-249.

Arteaga-Garibay, R. I., Gómez-Estrada, M. M., Martínez-Peña, M. D., Cadena-Zamudio, J. D., Avendaño-Arrazate, C. H. 2016. Functional metabolic diversity in microbial communities associated to rhizosphere soil of maize (Zea mays L.) amarillo-zamorano and jala cultivars. Agroproductividad. 9, 87-91.

Azarmi, R., Hajieghrari, B., Giglou, A. 2011. Effect of Trichoderma isolates on tomato seedling growth response and nutrient uptake. Afr. J. Biotechnol. $10,5850-5855$.

Björkman, T., Blanchard, L.M. and Harman, G.E. 1998. Growth enhancement of shrunken-2 sweet corn when colonized with Trichoderma harzianum 1295-22: effect of environmental stress. J. Am. Soc. Hort. Sci. 123, 35-40.
Bonner, J, Bandurski, R.S. 1952. Studies of the Physiology, Pharmacology, and Biochemistry of the Auxins. Annu. Rev. Plant Physiol. 3, 59-86.

Bric JM, RM Bostock, and SE Silverstone. 1991 Rapid in situ assay for indoleacetic acid production by bacteria immobilized on a nitrocellulose membrane. Applied and Environmental Microbiology. 57, 535-538.

Carvalho, D. D. C., Mello, S. C. M., Lobo Junior, M and Geraldine, A. M. 2011. Biocontrol of seed pathogens and growth promotion of common bean seedlings by Trichoderma harzianum. Pesq. Agropec. Bras. 46, 822-828.

Colla, G., Rouphael, Y., Di Mattia, E., El-Nakhel, C., Cardarelli, M. 2015. Co-inoculation of Glomus intraradices and Trichoderma atroviride acts as a biostimulant to promote growth, yield and nutrient uptake of vegetable crops. J. Sci. Food Agric. 95, 1706-1715.

Contreras-Cornejo, H.A., Macías-Rodríguez, L., Alfaro-Cuevas, R., López-Bucio, J. 2014. Trichoderma spp. improve growth of Arabidopsis seedlings under salt stress through enhanced root development, osmolite production, and $\mathrm{Na}^{+}$ elimination through root exudates. Mol. Plant Microbe Interact. 27, 503-514.

Contreras-Cornejo, H.A., Macías-Rodríguez, L., Herrera-Estrella, A., López-Bucio, J. 2014b. The 4-phosphopantetheinyl transferase of Trichoderma virens plays arole in plant protection against Botrytis cinerea through volatile organic compound emission. Plant Soil. 379, 261-274.

Gupta, H., Saini, R. V., Pagadala, V., Kumar, N., Sharma, D. K., Saini, A. K. 2016. Analysis of plant growth promoting potential of endophytes isolated from Echinacea purpurea and Lonicera japonica. J. Soil Sci. Plant Nutr. 16, 558-577. 
Hung, R., Lee, S., Bennett, J. W. 2013. Arabidopsis thaliana as a model system for testing the effect of Trichoderma volatile organic compounds. Fungal Ecol. 6, 19-26.

Kumar, K., Manigundan K and Amaresan, N. 2016. Influence of salt tolerant Trichoderma spp. on growth of maize (Altomare) under different salinity conditions. J. Basic Microbiol. 56, 1-10.

Lee, S., Yap, M., Behringer, G., Hung, R., Bennett, J. W. 2016. Volatile organic compounds emitted by Trichoderma species mediate plant growth. Fungal Biol. Biotechnol. 3:7

Lorito, M., Woo, S. L., Harman, G. E., Monte, E. 2010. Translational research on Trichoderma: from omics to the field. Annu. Rev. Phytopathol. 48, 395-417.

Madigan, M., Martinko, J., Parker, J. Brock biología de los microorganismos. (10 Ed). Madrid: Pearson Prentice Hall. 2003.

Martínez, O. A., Crowley, D. E., Mora, M. L., Jorquera, M. A. 2015. Short-term study shows that phytatemineralizing rhizobacteria inoculation affects the biomass, phosphorus (P) uptake and rhizosphere properties of cereal plants. J. Soil Sci. Plant Nutr. 15, 153-166.

Martínez-Peña, M. D., Lara-Aguilera, J., CadenaZamudio, J. D., Salinas-Moreno, Y., RamírezVega, H., Arteaga-Garibay, R. I. 2016. Rhizosphere microbiota from a mixed maize (Zea mays L.) crop in the Jalisco highlands: a descriptive study. Agroproductividad. 9, 65-70.

Mastouri, F., Björkman, T., Harman, G.E. 2010. Seed treatment with Trichoderma harzianum alleviates biotic, abiotic, and physiological stresses in germinating seeds and seedlings. Phytopathology. 100, 1213-1221.
Menezes-Blackburn, D., Inostroza, N.G., Gianfreda, L., Greiner, R., Mora, M.L., Jorquera, M.A. 2016. Phytase-producing Bacillus sp. inoculation increases phosphorus availability in cattle manure. J. Soil Sci. Plant Nutr. 16, 200-210.

Molla, A.H., Haque, M.M., Haque, M.A., Ilias, G.N.M., 2012. Trichoderma-enriched biofertilizer enhances production and nutritional quality of tomato (Lycopersicon esculentum Mill) and minimizes NPK fertilizer use. Agric. Res. 1, 265272.

Rajput, A.Q., Khanzada, M.A. and Shahzad, S. 2014a. Effect of different organic substrates and carbon and nitrogen sources on growth and shelf life of Trichoderma harzianum. J. Agr. Sci. Tech. 16, 731-745.

Rajput, A.Q., Khanzada, M.A. and Shahzad, S. 2014b. Effect of different substrates and carbon and nitrogen sources on growth and shelf life of Trichoderma pseudokoningii. Int. J. Agric. Biol. 16, 893- 898 .

Rubio, M.B., Hermosa, R., Vicente, R., GómezAcosta, F. A., Morcuende R., Monte E., Bettiol W. 2017. The combination of Trichoderma harzianum and chemical fertilization leads to the deregulation of phytohormone networking, preventing the adaptive responses of tomato plants to salt stress. Front. Plant Sci. 8, 294.

Rubio, M.B., Quijada, N.M., Pérez, E., Domínguez, S., Monte, E., Hermosa, R. 2014. Identifying beneficial qualities of Trichoderma parareesei for plants. Appl. Environ. Microbiol. 80, 1864-1873.

Saravanakumar, K., Shanmuga, A.V., Kathiresan, K. 2013. Effect of Trichoderma on soil phosphate solubilization and growth improvement of Avicennia marina. Aquat. Bot. 104, 101-105. 
Schoch, C.L, Seifert K.A, Huhndorf S., Robert V., Spouge J.L., Levesque A.C., Chen W. 2012. Nuclear ribosomal internal transcribed spacer (ITS) region as a universal DNA barcode marker for Fungi. PNAS. 109, 6241-6246.

Studholme, D.J., Harris, B., Le Cocq, K., Winsbury, R., Perer, A.V., Ryder, L., Ward, J. L., Beale, M.H., Thornton, C.R., Grant, M. 2013. Investigating the beneficial traits of Trichoderma hamatum GD 12 for sustainable agriculture-insights from genomics. Front. Plant Sci. 4, 1-13.

Tucci, M., Ruocco, M., De Masi, L., De Palma, M., Lorito, M. 2011. The beneficial effect of Trichoderma spp. on tomato is modulated by the plant genotype. Mol. Plant Pathol. 12, 341-354.

Vanneste, S., Friml, J. 2009. Auxin: a trigger for change in plant development. Cell. 136, 10051016
Vargas, W. A., Mandawe, J. C and Kenerley, C. M. 2009. Plant-derived sucrose is a key element in the symbiotic association between Trichoderma virens and maize plants. Plant Physiol. 151, 792808.

Vinale, F., Sivasithamparam, K., Ghisalberti, E.L., Marra, R., Barbetti, M.J., Li, H., Woo S.L., Lorito, M. 2008. A novel role for Trichoderma secondary metabolites in the interactions with plants. Physiol. Mol. Plant Pathol. 72, 80-86.

Vos, C. M. F., De Cremer, K., Cammue, B. P. A., De Coninck, B. 2015. The toolbox of Trichoderma spp. in biocontrol of Botrytis cinerea disease. Mol. Plant Pathol. 16, 400-412.

Zhao, L., Wang, F., Zhang, Y., Zhang, J. 2014. Involvement of Trichoderma asperellum strain T6 in regulating iron acquisition in plants. J. Basic Microbiol. 54, 115-124. 